\title{
Variations in 30-day readmissions and length of stay among spine surgeons: a national study of elective spine surgery among US Medicare beneficiaries
}

\author{
Siddhartha Singh, MD, MS, ${ }^{1}$ Rodney Sparapani, $\mathrm{PhD},{ }^{2}$ and Marjorie C. Wang, MD, $\mathrm{MPH}^{3}$ \\ Departments of ${ }^{1}$ Medicine, ${ }^{2 B}$ Biostatistics, and ${ }^{3}$ Neurosurgery, Medical College of Wisconsin, Milwaukee, Wisconsin
}

OBJECTIVE Pay-for-performance programs are targeting hospital readmissions. These programs have an underlying assumption that readmissions are due to provider practice patterns that can be modified by a reduction in reimbursement. However, there are limited data to support the role of providers in influencing readmissions. To study this, the authors examined variations in readmission rates by spine surgeon within 30 days among Medicare beneficiaries undergoing elective lumbar spine surgery for degenerative conditions.

METHODS The authors applied validated ICD-9-CM algorithms to 2003-2007 Medicare data to select beneficiaries undergoing elective inpatient lumbar spine surgery for degenerative conditions. Mixed models, adjusting for patient demographics, comorbidities, and surgery type, were used to estimate risk of 30-day readmission by the surgeon. Length of stay (LOS) was also studied using these same models.

RESULTS A total of 39,884 beneficiaries were operated on by 3987 spine surgeons. The mean readmission rate was $7.2 \%$. The mean LOS was 3.1 days. After adjusting for patient characteristics and surgery type, 1 surgeon had readmission rates significantly below the mean, and only 5 surgeons had readmission rates significantly above the mean. In contrast, for LOS, the patients of 288 surgeons $(7.2 \%)$ had LOS significantly lower than the mean, and the patients of 397 surgeons $(10.0 \%)$ had LOS significantly above the mean. These findings were robust to adjustments for surgeon characteristics and clustering by hospital. Similarly, hospital characteristics were not significantly associated with readmission rates, but LOS was associated with hospital for-profit status and size.

CONCLUSIONS The authors found almost no variations in readmission rates by surgeon. These findings suggest that surgeon practice patterns do not affect the risk of readmission. Likewise, no significant variation in readmission rates by hospital characteristics were found. Strategies to reduce readmissions would be better targeted at factors other than providers.

https://thejns.org/doi/abs/10.3171/2018.1.SPINE171064

KEYWORDS 30-day readmissions; length of stay; spine surgery

$\mathrm{R}$ EADMISSIONS for Medicare beneficiaries within 30 days of discharge are frequent and costly. ${ }^{17}$ Some readmissions may represent poor quality of care, ${ }^{16}$ and the risk of readmission varies among hospitals, suggesting that reduction in readmissions is possible. ${ }^{17}$ Therefore, both private payers and the Centers for Medicare and Medicaid Services (CMS) have initiated programs aimed at reducing readmissions. Some of these programs, such as the CMS Hospital Readmission Reduction Program
(HRRP), hold hospitals accountable for reducing readmissions among the medical and surgical patients they discharge.$^{8}$ Hospitals may transfer this accountability to their physicians. However, physicians are also increasingly being held directly accountable for readmissions. For example, the CMS Quality Payment Program (QPP) holds physicians accountable for their patients' readmissions, ${ }^{7}$ as does public reporting of quality data by individual physicians. ${ }^{4}$ Despite this, it is unclear if placing accountability

ABBREVIATIONS CMS = Centers for Medicare and Medicaid Services; GLMM = generalized linear mixed model; LOS = length of stay; MEDPAR = Medicare Provider Analysis and Review.

SUBMITTED September 28, 2017. ACCEPTED January 11, 2018.

INCLUDE WHEN CITING Published online June 1, 2018; DOI: 10.3171/2018.1.SPINE171064. 
for readmissions on physicians is justified, because there are limited data to show that physician practice patterns influence readmissions.

The purpose of this study was to evaluate the role of physicians, and specifically spine surgeons, in readmission rates after elective lumbar spine surgery for degenerative conditions in a national study of US Medicare beneficiaries. Because variations in length of stay (LOS) have been shown to be associated with surgeon practice patterns in other populations, we also evaluated the variation in LOS in the same Medicare cohort. ${ }^{15}$

\section{Methods}

\section{Study Population and Data Source}

Medicare claims were provided by CMS, including Medicare Provider Analysis and Review (MEDPAR) hospital claims, outpatient clinic claims, carrier physician claims, and Denominator enrollment files, which were linked by beneficiary identifier and date from 2003 to 2007. This study was approved by our institutional review board. We used published and validated ICD-9-CM algorithms to select patients who underwent elective nonurgent lumbar spine surgery for degenerative conditions such as lumbar stenosis in the lumbosacral spine. ${ }^{8,16,17}$ Lumbar fusion and nonfusion procedures were included.

We excluded beneficiaries who were enrolled in a Health Maintenance Organization because some of their claims may not have been in the Medicare database. ${ }^{30} \mathrm{In}$ addition, we required that all beneficiaries be continuously enrolled in Medicare from 1 year before the index surgical admission to 30 days after discharge in order to capture all relevant codes. Since reoperations could be associated with our outcomes, we excluded beneficiaries whose index admission codes indicated a reoperation. Because of the lack of sensitivity of the reoperation codes, ${ }^{8}$ we used the year prior to the index surgical admission as a "washout period" and excluded any beneficiaries who underwent spine surgery within the year prior to the index qualifying surgery.

\section{Patient Characteristics}

We used MEDPAR, Denominator, and Vital Status files to determine patient characteristics, including race or ethnicity, census region, and dual Medicare and state buy-in Medicaid eligibility. Comorbid conditions were identified using ICD-9-CM diagnosis codes associated with the index hospital admission. Comorbid conditions were defined according to Deyo et al.' $\mathrm{s}^{10}$ adaptation of the Charlson Comorbidity Index. ${ }^{9}$

\section{Type of Surgery and Surgical Approach}

Type of surgery and surgical approach were defined using the published and validated ICD-9-CM algorithms for lumbar spine. ${ }^{8,16,17}$ For fusion procedures, the number of levels fused was further stratified using ICD-9-CM procedure codes. ${ }^{5,12}$ Fusion procedures were categorized in 3 ways: 1) nonfusion procedure (baseline for comparison); 2) fusion, 1-3 levels; or 3) fusion, $\geq 4$ levels. All surgical procedures were performed from a posterior approach. Although the discectomy code (ICD-9-CM 80.51) does not specify the approach, when this code was used alone in the lumbosacral spine (without any other procedure code denoting surgical approach), the procedure was classified as posterior.

\section{Surgeon and Hospital Characteristics}

Surgeon characteristics were obtained from the American Medical Association's Physician Professional Database. Surgeons were included if they were orthopedic surgeons or neurosurgeons and had hospital claims associated with the admission for lumbar spine surgery in our defined cohort. Year of training was defined as years from completing residency to the hospital admission. Surgeon volume was defined solely on the number of elective lumbar spine surgery cases for degenerative conditions per year among Medicare beneficiaries. Hospital characteristics were obtained from the American Hospital Association's hospital survey. Hospital characteristics included hospital volume of elective lumbar spine surgeries per year, hospital size (number of beds), hospital for-profit status, and Council of Teaching Hospitals affiliation.

\section{Thirty-Day Readmissions and Reasons for Readmissions}

Thirty-day readmissions were defined as all readmissions to a short-term acute care hospital within 30 days of discharge among beneficiaries who were discharged alive from the index admission. Readmissions for rehabilitation were excluded (ICD-9-CM code V57). Because 30-day readmissions are currently counted after an inpatient hospital stay, all included surgeries were inpatient admissions.

\section{Length of Stay}

The LOS was defined as the number of days from the index hospital admission to the index hospital discharge.

\section{Data Analysis}

We chose a modeling method that would address the inferential needs presented by the data we collected and the question at hand. Generalized linear mixed models (GLMMs) are well suited for this investigation. First, GLMMs support both dichotomous outcomes (for 30-day readmissions) and count outcomes (for LOS) by what we call the logistic mixed model (LogMM) and the Poisson mixed model (PoiMM), respectively. Second, GLMMs handle clustered outcomes which, in this case, are due to the lack of independence between patients treated at the same hospital and/or by the same surgeon.

For each outcome, we fit a series of models of increasing complexity, which helps us assess the effect of patient and treatment characteristics as well as the relative magnitude of surgeon and hospital characteristics and clustering variation. We fit the first model with clustering for surgeon and an intercept only; i.e., no covariates. We fit the second model with clustering for surgeon and adjusting for the following covariates: age, sex, race, dual Medicare and Medicaid enrollment, census division, Charlson-Deyo Comorbidity Index, and surgery. We fit a third model with clustering for surgeon and hospital, including the same covariates as the second model. Finally, we fit a fourth model with clustering for surgeon and hospital, includ- 
TABLE 1. Characteristics in 39,884 patients with elective lumbar spine surgery

\begin{tabular}{lcc}
\hline \multicolumn{1}{c}{ Characteristic } & No. & Percent \\
\hline Age (yrs) & & \\
\hline $66-70$ & 12,808 & 32.1 \\
\hline $71-75$ & 11,595 & 29.1 \\
\hline $76-80$ & 9,317 & 23.4 \\
\hline $81-90$ & 6,164 & 15.5 \\
\hline Female sex & 22,329 & 56.0 \\
\hline Race & & \\
\hline White & 37,661 & 94.5 \\
\hline Black & 1,274 & 3.2 \\
\hline Other & 949 & 2.3 \\
\hline Medicaid eligible & 2,181 & 5.5 \\
\hline Comorbidities, Charlson-Deyo Index & & \\
\hline 0 & 22,326 & 56.0 \\
\hline 1 & 12,252 & 30.7 \\
\hline$\geq 2$ & 5,306 & 13.3 \\
\hline Readmitted w/in 30 days & 2,884 & 7.2 \\
\hline Median LOS in days (IQR) & $3.0(1.0-4.0)$ \\
\hline
\end{tabular}

ing the same covariates as the second and third models, plus surgeon volume, surgeon specialty, surgeon years of training, hospital volume, number of hospital beds, hospital for-profit status, and hospital medical school affiliation (Council of Teaching Hospitals affiliation). We used the second model for our results, and we used the third and fourth models for sensitivity analysis.

\section{Results}

Between 2004 and 2007, 39,884 Medicare beneficiaries underwent elective lumbar surgeries for degenerative conditions. These surgeries were performed by 3987 spine surgeons (orthopedic surgeons and neurosurgeons). Patient characteristics are listed in Table 1. The majority of the cohort was female (56\%) and white $(94.5 \%)$. A total of $5.5 \%$ had joint Medicare/Medicaid status, which is an indicator of low socioeconomic status. The majority of the cohort was healthy; $13.3 \%$ had $\geq 2$ comorbidities.

Type of surgery, surgeon characteristics, and hospital characteristics are listed in Table 2. Overall, 60.8\% of surgeons were neurosurgeons. Surgeons were divided into tertiles based on years of training and number of cases per year $(<12,12-24$, and $>24$ Medicare elective lumbar surgeries per year). The majority of hospitals had notfor-profit status. Hospitals were also divided into tertiles based on hospital volume of Medicare cases per year. Hospital size ranged from $<100$ beds to $\geq 300$ beds. Approximately one-third of the hospitals were 100-199 beds in size. Just over half of hospitals were affiliated with medical schools $(53.0 \%)$.

\section{Thirty-Day Readmissions}

The mean 30-day readmission rate was 7.2\%. After adjusting for patient characteristics and type of surgery, 1
TABLE 2. Type of surgery, surgeon characteristics, and hospital characteristics in 39,884 patients with elective lumbar spine surgery

\begin{tabular}{|c|c|c|}
\hline Variable & No. & Percent \\
\hline \multicolumn{3}{|l|}{ Type of surgery } \\
\hline \multicolumn{3}{|l|}{ Levels fused } \\
\hline None & 23,257 & 58.3 \\
\hline 1-3 levels & 14,041 & 35.2 \\
\hline$\geq 4$ levels & 2,586 & 6.5 \\
\hline \multicolumn{3}{|l|}{ Surgeon and hospital characteristics } \\
\hline \multicolumn{3}{|l|}{ Surgeon vol of Medicare lumbar cases per yr } \\
\hline$<12$ & 10,370 & 26.0 \\
\hline $12-24$ & 14,089 & 35.3 \\
\hline$>24$ & 15,425 & 38.7 \\
\hline \multicolumn{3}{|l|}{ Surgeon yr of training } \\
\hline $1956-1988$ & 13,043 & 33.5 \\
\hline 1989-1996 & 12,803 & 32.9 \\
\hline 1997 or later & 13,039 & 33.5 \\
\hline \multicolumn{3}{|l|}{ Surgeon specialty } \\
\hline Neurosurgeon & 24,246 & 60.8 \\
\hline Orthopedic surgeon & 15,638 & 39.2 \\
\hline \multicolumn{3}{|c|}{ Hospital vol of Medicare lumbar cases per yr } \\
\hline$<40$ & 12,912 & 32.4 \\
\hline $40-79$ & 14,644 & 36.7 \\
\hline$\geq 80$ & 12,328 & 30.9 \\
\hline \multicolumn{3}{|l|}{ Hospital size (no. of beds) } \\
\hline $1-99$ & 7,566 & 19.2 \\
\hline $100-199$ & 12,989 & 32.9 \\
\hline $200-299$ & 9,113 & 23.1 \\
\hline$\geq 300$ & 9,768 & 24.8 \\
\hline \multicolumn{3}{|l|}{ Hospital for-profit status } \\
\hline For profit & 5,989 & 15.2 \\
\hline Not for profit & 30,394 & 76.9 \\
\hline Government & 3,134 & 7.9 \\
\hline Council of Teaching Hospitals affiliation & 20,928 & 53.0 \\
\hline
\end{tabular}

surgeon had a 30-day readmission rate below the mean and only 5 surgeons had a readmission rate significantly above the mean (Fig. 1). Our findings were robust to adjustments for surgeon characteristics and clustering within hospitals. Hospital characteristics (volume, size, for-profit status, and medical school affiliation) were not significantly associated with 30-day readmission rates.

\section{Length of Stay}

The mean LOS was 3.1 days (SD 2.2). The median LOS was 3.0 days (IQR 1.0-4.0 days). Among those who were readmitted within 30 days, the mean LOS was 3.8 days (SD 3.0, median 3.0 days, IQR 3.0). There was no significant correlation between LOS and 30-day readmission (Spearman's rank correlation coefficient 0.1).

After adjusting for patient characteristics and type of surgery, we noted significant variations in LOS by sur- 


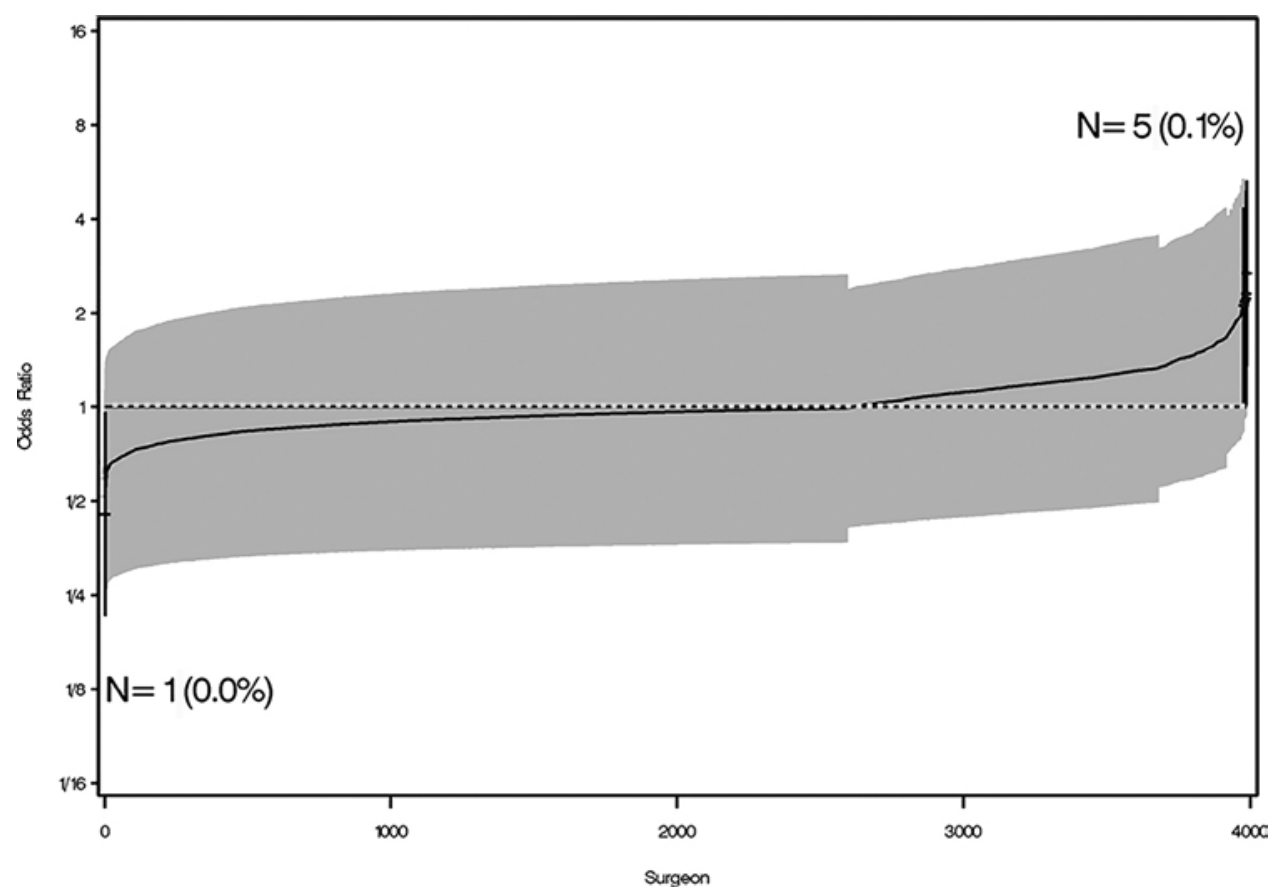

FIG. 1. Variation in risk of 30-day readmission by spine surgeon. The dotted line represents an odds ratio of 1. Each individual spine surgeon's estimates of risk of readmission are presented as vertical bars (gray and black). The black line connects the mean estimated odds ratio for each spine surgeon, and the height of the bars represents the upper and lower $95 \%$ confidence intervals. The black bars represent spine surgeons who have 95\% confidence intervals for the odds of readmission within 30 days that are above or below 1.

geon. We found that $7.2 \%$ of surgeons $(n=288)$ had significantly shorter LOS and $10 \%$ had significantly longer LOS $(\mathrm{n}=397)$ than the mean (Fig. 2). In addition, we found significant variation in LOS by hospital for-profit status and size, with for-profit hospital status associated with shorter LOS $(p=0.005)$, but larger hospital size associated with longer LOS $(\mathrm{p}<0.001)$.

\section{Discussion}

Among our cohort of 39,884 Medicare beneficiaries undergoing elective lumbar spine surgeries, we found no significant variation in readmission rates by spine surgeon. This suggests that individual surgeon practice patterns do not significantly impact the risk of readmission, and is consistent with prior research that showed that physicians do not impact risk of readmission for medical inpatients and that patient factors contribute the most to the risk of readmission. ${ }^{11,15}$ In addition, studies of unplanned readmissions after spine surgery have shown significant associations with patient race, comorbid conditions, preoperative diagnoses, and complications after surgical procedures. ${ }^{1-3,13,19,21,23,26,29}$ Variations by spine surgeon specialty have been studied, but no significant differences were noted between orthopedic and neurological surgeons in spine surgery. ${ }^{22,24,25}$ Our findings suggest that patient characteristics may be important factors influencing readmission rates. Programs that target physicians to reduce readmission rates may have limited effectiveness.

In contrast, we found significant variations in LOS by spine surgeons, even after adjustment for patient and hos- pital factors. We found no correlation between LOS and readmission rates. This is also consistent with previous research, and points to surgical practice patterns contributing to LOS. ${ }^{15,28}$ Goodwin et al. and Krell et al. separately showed that LOS for hospitalized patients varies by physician, even after adjustment for patient factors. ${ }^{14,20}$ Caminiti et al. then showed that LOS could be reduced by placing accountability on physicians. ${ }^{6}$ As hospitals try to meet the challenge of providing more care in the face of declining reimbursement, ${ }^{27}$ our findings represent an opportunity for spine surgeons to impact the cost of care by decreasing variation in their clinical practice to also decrease LOS. Our finding of no significant correlation between LOS and readmission rates has also been described before among medical readmissions, ${ }^{18}$ and is reassuring because quality improvement to reduce LOS would be unlikely to have the unintended consequence of increasing readmission rates.

We found similar findings in studying hospital characteristics. Hospital volume of lumbar spine surgery, size, for-profit status, and medical school affiliation were not significantly associated with readmission rates. However, size and for-profit status were significantly associated with LOS.

Our study has limitations. It is an observational study and susceptible to bias and confounding. We studied Medicare beneficiaries; our findings may not apply to a younger population. Our study is limited by use of ICD9-CM codes and their lack of detailed descriptions of surgical procedures, but we limited our sample to elective lumbar spine surgeries only, and stratified the sample by 


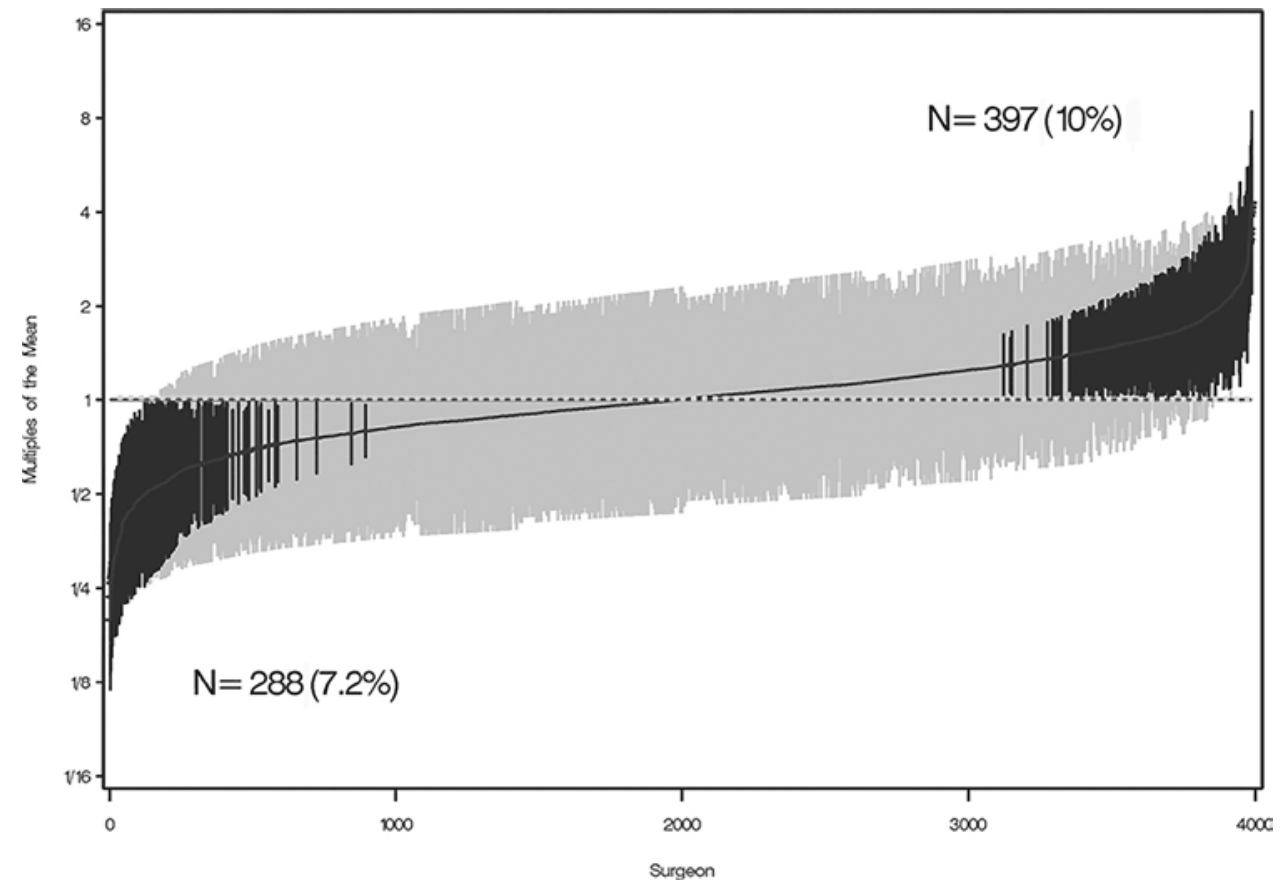

FIG. 2. Variation in LOS by spine surgeon. The dotted line represents the average LOS normalized to a value of 1. Each individual spine surgeon's estimates of LOS in multiples of the mean are presented as vertical bars (gray and black). The black line connects the mean estimated LOS for each spine surgeon, and the height of the bars represents the upper and lower $95 \%$ confidence intervals. The black bars represent spine surgeons who have 95\% confidence intervals for the LOS that are above or below the normalized average of 1 .

the number of levels fused. Surgeon volume could only be defined using elective lumbar spine surgeries for degenerative conditions, but spine surgeons would typically perform surgery in other levels of the spine as well. This data set did not include the number of spine surgeries performed in all populations-for example, among patients with private insurance or younger patients. Spine surgery volume for urgent spine conditions was not counted. Hospital characteristics were also limited to those included in the data set, and other factors may play a role in these outcomes. Despite these limitations, use of this Medicare data set allowed us to follow patients longitudinally to determine the 30-day readmission rates. Our study data precedes major policy changes impacting readmissions, such as the Hospital Readmission Reduction Program, but is still relevant because readmissions have been a persistent issue.

\section{Conclusions}

Our study shows that individual spine surgeons did not significantly influence variations in 30-day readmission rates, after adjusting for patient and hospital characteristics. However, similar to other studies of hospital LOS, individual spine surgeons did significantly influence variations in LOS. Our findings suggest that efforts to reduce readmission rates targeted at individual spine surgeons may have limited effectiveness.

\section{References}

1. Abt NB, McCutcheon BA, Kerezoudis P, Murphy M, Rinaldo
L, Fogelson J, et al: Discharge to a rehabilitation facility is associated with decreased 30-day readmission in elective spinal surgery. J Clin Neurosci 36:37-42, 2017

2. Adogwa O, Elsamadicy AA, Han JL, Karikari IO, Cheng J, Bagley CA: 30-day re-admission after spine surgery: an analysis of 1400 consecutive spine surgery patients. Spine (Phila Pa 1976) 42:520-524, 2017

3. Adogwa O, Elsamadicy AA, Mehta AI, Cheng J, Bagley CA, Karikari IO: Racial disparities in 30-day readmission rates after elective spine surgery: a single institutional experience. Spine (Phila Pa 1976) 41:1677-1682, 2016

4. Allen M, Pierce O: Making the cut. Why choosing the right surgeon matters even more than you know. ProPublica. July 13, 2015. (https://www.propublica.org/article/surgery-riskspatient-safety-surgeon-matters) [Accessed February 15, 2018]

5. Cahill KS, Chi JH, Day A, Claus EB: Prevalence, complications, and hospital charges associated with use of bonemorphogenetic proteins in spinal fusion procedures. JAMA 302:58-66, 2009

6. Caminiti C, Meschi T, Braglia L, Diodati F, Iezzi E, Marcomini B, et al: Reducing unnecessary hospital days to improve quality of care through physician accountability: a cluster randomised trial. BMC Health Serv Res 13:14, 2013

7. Centers for Medicare and Medicaid Services: MACRA: MIPS \& APMs. CMS.gov. (https://www.cms.gov/medicare/ quality-initiatives-patient-assessment-instruments/valuebased-programs/macra-mips-and-apms/macra-mips-andapms.html) [Accessed February 15, 2018]

8. Centers for Medicare and Medicaid Services: Readmissions Reduction Program (HRRP). CMS.gov. (https://www. cms.gov/Medicare/Medicare-Fee-for-Service-Payment/ AcuteInpatientPPS/Readmissions-Reduction-Program.html) [Accessed February 15, 2018]

9. Charlson ME, Pompei P, Ales KL, MacKenzie CR: A new method of classifying prognostic comorbidity in longitudinal 
studies: development and validation. J Chronic Dis 40:373383, 1987

10. Deyo RA, Cherkin DC, Ciol MA: Adapting a clinical comorbidity index for use with ICD-9-CM administrative databases. J Clin Epidemiol 45:613-619, 1992

11. Deyo RA, Ching A, Matsen L, Martin BI, Kreuter W, Jarvik $\mathrm{JG}$, et al: Use of bone morphogenetic proteins in spinal fusion surgery for older adults with lumbar stenosis: trends, complications, repeat surgery, and charges. Spine (Phila Pa 1976) 37:222-230, 2012

12. Deyo RA, Mirza SK, Martin BI, Kreuter W, Goodman DC, Jarvik JG: Trends, major medical complications, and charges associated with surgery for lumbar spinal stenosis in older adults. JAMA 303:1259-1265, 2010

13. Garcia RM, Choy W, DiDomenico JD, Barrington N, Dahdaleh NS, Rodriguez HE, et al: Thirty-day readmission rate and risk factors for patients undergoing single level elective anterior lumbar interbody fusion (ALIF). J Clin Neurosci 32:104-108, 2016

14. Goodwin JS, Lin YL, Singh S, Kuo YF: Variation in length of stay and outcomes among hospitalized patients attributable to hospitals and hospitalists. J Gen Intern Med 28:370-376, 2013

15. Gross TS, McCracken C, Heiss KF, Wulkan ML, Raval MV: The contribution of practice variation to length of stay for children with perforated appendicitis. J Pediatr Surg 51:1292-1297, 2016

16. Halfon P, Eggli Y, Prêtre-Rohrbach I, Meylan D, Marazzi A, Burnand B: Validation of the potentially avoidable hospital readmission rate as a routine indicator of the quality of hospital care. Med Care 44:972-981, 2006

17. Jencks SF, Williams MV, Coleman EA: Rehospitalizations among patients in the Medicare fee-for-service program. $\mathbf{N}$ Engl J Med 360:1418-1428, 2009

18. Kaboli PJ, Go JT, Hockenberry J, Glasgow JM, Johnson SR, Rosenthal GE, et al: Associations between reduced hospital length of stay and 30-day readmission rate and mortality: 14-year experience in 129 Veterans Affairs hospitals. Ann Intern Med 157:837-845, 2012

19. Khanna R, McDevitt JL, McClendon J Jr, Smith ZA, Dahdaleh NS, Fessler RG: Utility of readmission rates as a quality of care measure and predictors of readmission within 30 days after spinal surgery: a single-center, multivariate analysis. Spine (Phila Pa 1976) 40:1769-1774, 2015

20. Krell RW, Girotti ME, Dimick JB: Extended length of stay after surgery: complications, inefficient practice, or sick patients? JAMA Surg 149:815-820, 2014

21. Lee NJ, Kothari P, Phan K, Shin JI, Cutler HS, Lakomkin N, et al: The incidence and risk factors for 30-day unplanned readmissions after elective posterior lumbar fusion. Spine (Phila Pa 1976) 43:41-48, 2016

22. Mabud T, Norden J, Veeravagu A, Swinney C, Cole T, McCutcheon BA, et al: Complications, readmissions, and revisions for spine procedures performed by orthopedic surgeons versus neurosurgeons: a retrospective, longitudinal study. Clin Spine Surg 30:E1376-E1381, 2017

23. Martin J, Wang TY, Loriaux DB, Desai R, Adogwa O, Kuchibhatla M, et al: Race as a predictor of postoperative hospital readmission after spine surgery. Neurosurgery 63 (Suppl 1):191, 2016 (Abstract)

24. McCutcheon BA, Ciacci JD, Marcus LP, Noorbakhsh A, Gonda DD, McCafferty R, et al: Thirty-day perioperative outcomes in spinal fusion by specialty within the NSQIP database. Spine (Phila Pa 1976) 40:1122-1131, 2015

25. Seicean A, Alan N, Seicean S, Neuhauser D, Benzel EC, Weil RJ: Surgeon specialty and outcomes after elective spine surgery. Spine (Phila Pa 1976) 39:1605-1613, 2014

26. Su AW, Habermann EB, Thomsen KM, Milbrandt TA, Nassr A, Larson AN: Risk factors for 30-day unplanned readmission and major perioperative complications after spine fusion surgery in adults: a review of the National Surgical Quality Improvement Program Database. Spine (Phila Pa 1976) 41:1523-1534, 2016

27. Wang MC, Kreuter W, Wolfla CE, Maiman DJ, Deyo RA: Trends and variations in cervical spine surgery in the United States: Medicare beneficiaries, 1992 to 2005. Spine (Phila Pa 1976) 34:955-963, 2009

28. Wang MC, Laud PW, Macias M, Nattinger AB: Strengths and limitations of International Classification of Disease Ninth Revision Clinical Modification codes in defining cervical spine surgery. Spine (Phila Pa 1976) 36:E38-E44, 2011

29. Wang MC, Shivakoti M, Sparapani RA, Guo C, Laud PW, Nattinger AB: Thirty-day readmissions after elective spine surgery for degenerative conditions among US Medicare beneficiaries. Spine J 12:902-911, 2012

30. Wennberg JE: Time to tackle unwarranted variations in practice. BMJ 342:d1513, 2011

\section{Disclosures}

Dr. Singh is a consultant for AstraZeneca. This study was funded in part by the Robert Wood Johnson Foundation (RWJF) Physician Faculty Scholars Program and by the Department of Neurosurgery, Medical College of Wisconsin, research and salary support (Dr. Wang). Dr. Sparapani received salary support from the RWJF.

\section{Author Contributions}

Conception and design: Wang, Singh. Acquisition of data: Wang, Sparapani. Analysis and interpretation of data: all authors. Drafting the article: all authors. Critically revising the article: all authors. Reviewed submitted version of manuscript: all authors. Approved the final version of the manuscript on behalf of all authors: Wang. Statistical analysis: all authors. Administrative/ technical/material support: Wang. Study supervision: Wang.

\section{Supplemental Information}

Previous Presentations

This study was presented at the CNS Annual Meeting in New Orleans, Louisiana (oral presentation), 2015.

\section{Correspondence}

Marjorie C. Wang: Medical College of Wisconsin, Milwaukee, WI. mwang@mcw.edu. 